\title{
Nikon.
}

\section{Application of patterned illumination using a DMD for optogenetic control of signaling}

\author{
Digital micromirror devices (DMDs) are powerful tools for photostimulation applications, including \\ photoconversion and optogenetic manipulation, owing to their robust ability to produce novel \\ illumination patterns with high spatiotemporal resolution. In this Application Note we showcase \\ recent work describing how DMD technology integrated into a Nikon system can be applied toward \\ light-gated optogenetic control of intracellular signaling.
}

DMD technology and integration into imaging experiments DMDs consist of rectangular arrays of many thousands to millions of microscopic mirrors (Fig. 1), each of which may be deflected between 'on' and 'off' positions separated by an $\sim 12^{\circ}$ tilt. As each mirror may be independently controlled, countless programmable illumination patterns are possible. Furthermore, DMDs are compatible with LED light sources, providing flexibility and cost savings compared to alternative laser-based systems for photostimulation, such as galvo scanners.
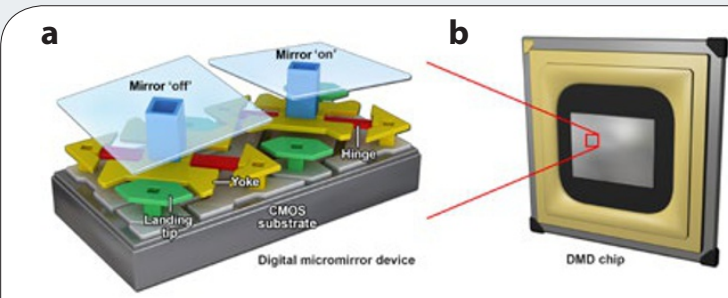

Figure 1 | Digital micromirror device (DMD) construction. (a) An illustration of a pair of micromirrors, with one deflected in the 'off' direction and the other in the 'on' direction. Each micromirror is attached to a yoke, which is in turn connected to support posts connecting the assembly to the underlying complementary metal-oxide semiconductor (CMOS) substrate. The landing tips specify the end of the travel range. Mirrors on the Polygon400 DMD are $18.0 \mu \mathrm{m}$ on each edge, and the entire chip has a projection area of $8.7 \times 15.5 \mathrm{~mm}$ on Nikon microscopes. Near-diffraction-limited pixel sizes can be realized with a variety of objectives, allowing for stimulation with intracellular specificity. (b) A'zoomedout'illustration of an entire DMD.

With Nikon's Laser Applications (LAPP) illuminator system, up to two DMDs (depending on the model) may be added to the microscope light path, allowing users to simultaneously stimulate with two sets

\section{John Allen}

Nikon Instruments, Inc., Melville, New York, USA. Correspondence should be addressed to J.A. (jrallen@nikon.net) or Lynne Chang (Ichang@nikon.net) of distinct patterns having different wavelengths and intensities. Furthermore, the LAPP system allows for up to five illumination modules on a single microscope, enabling easy combination of different imaging modalities such as DMD and total internal reflection fluorescence (TIRF) (Fig. 2). Because LAPP illuminators attach to the back port of the microscope, users can add further imaging functionality through the side port(s), including confocal scanners. The LAPP system also provides a built-in upgrade path. For example, users may start with only an epifluorescence module and then easily add further functionality as needs evolve.

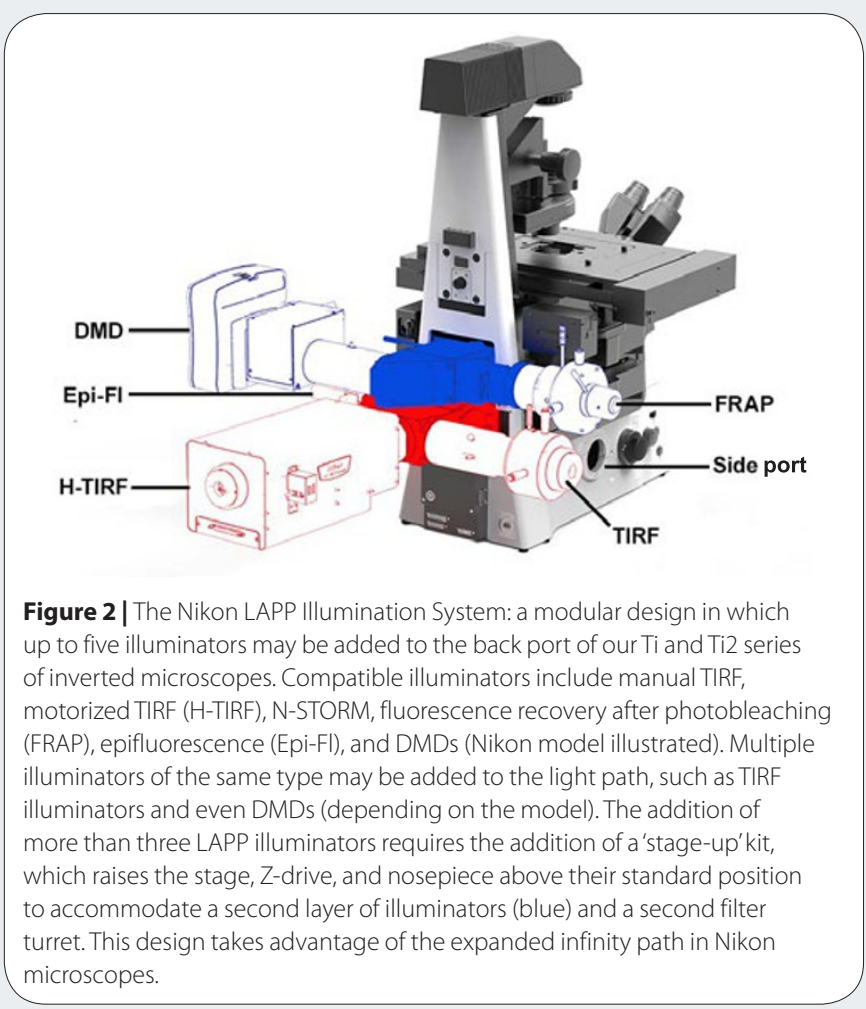


The ease of use of DMD modules is maximized via integration in Nikon's NIS-Elements software. Calibration between DMD mirrors and camera pixels is easy and automatic, and DMD-controlled spatial patterns can be simply drawn on images acquired from the camera. Moreover, NIS-Elements supports hardware triggering of DMDs and custom control through the Illumination Sequence module, where users may program ultra-fast hardware-triggered multi-wavelength imaging sequences, ideal for live-cell experimentation. Switching between globally distinct DMD illumination patterns is fast-up to $4,000 \mathrm{~Hz}$ depending on the number of patterns-and supports variable light output from triggered LED sources. The generation of a series of changing illumination patterns in an experiment is easily achievable.

\section{Optogenetic control of signaling with a DMD}

DMD technology benefits many optogenetics-based experimental systems, famously including the stimulation of neurons that express opsin constructs. Here we examine the utility of DMDs in experimental systems that use light-gated control of plasma membrane recruitment, as exemplified by the OptoSOS system developed by Johnson et al. ${ }^{1}$ for probing the role of the Ras-Erk signaling pathway in the developing Drosophila embryo.

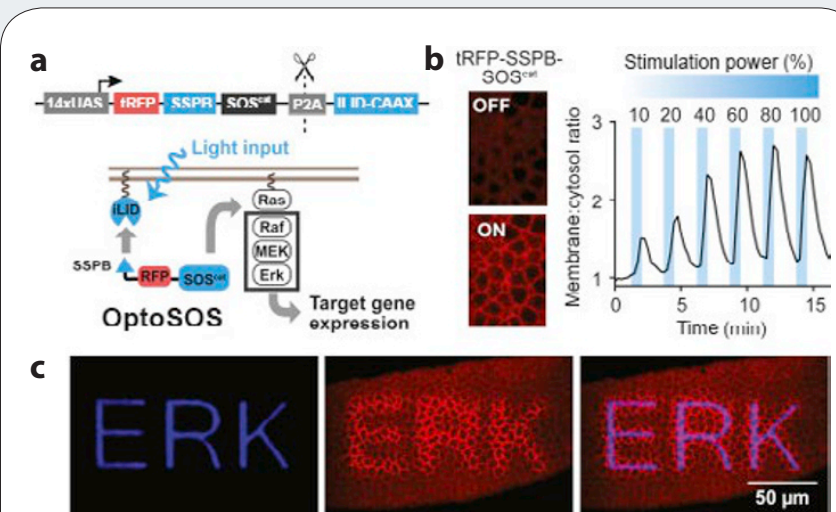

Figure $\mathbf{3}$ | Using a DMD to drive the recruitment of catalytically active SOS protein (SOS ${ }^{\text {cat }}$ ) to plasma membranes in select regions of a developing embryo, thus resulting in downstream activation of Erk signaling. (a) tRFPSSPB-SOS ${ }^{\text {cat }}$ and iLID-CAAX are expressed throughout the embryo. iLID-CAAX anchors in the plasma membrane and, upon stimulation with blue light, photodimerizes with SSPB, recruiting SOS ${ }^{c a t}$ and initiating the Ras-Erk signaling cascade. (b) The dependence of tRFP-SSPB-SOScat recruitment on stimulation power. (c) Left, the illumination pattern produced with a 450-nm blue LED (X-Cite XLED) and Polygon400 DMD. Middle, recruitment localization of tRFPSSPB-SOS ${ }^{\text {cat }}$ to plasma membranes in stimulated regions. Right, an overlay of the stimulation pattern and tRFP fluorescence. Reprinted with permission from ref. 1 .

Figure 3 illustrates how patterned illumination from a DMD can be used to control the spatial extent and magnitude of Erk signaling in a developing Drosophila embryo. Importantly, such a generalizable system can be extended to probe the function of other signaling pathways and/or other model organisms.

Previously, targeted expression systems and conditional mutants were the methods of choice for probing the role of signaling pathways in embryonic development. However, gain-of-function (GOF) pathway mutants can be problematic, exhibiting a limited spatial range of increased signaling and often resulting in complete lethality to the embryo. GOF mutants generally lack the 'on-off' switching capability of a system such as OptoSOS, which means that signaling cannot be stimulated at defined time points during development.

More recently, the work of Johnson et al. has been expanded to include the monitoring of immediate-early gene transcript and protein levels ${ }^{2}$. For this work, the PHY-PIF system was applied for optogenetic activation of Ras. PHY_PIF dimerization is induced by $650-\mathrm{nm}$ light and turned off by 750-nm light. In this instance two DMD modules (Polygon400, Mightex Systems (distributed by Nikon Instruments, Inc.)) were used: one for 650-nm activation, and one for 750-nm deactivation. This method yields richer data, enabling true quantitative evaluation of the fine balance between signaling and gene expression, and opening a number of new experimental avenues in the field.

1. Johnson, H.E. et al. Dev. Cell 40, 185-192 (2017).

2. Wilson, M.Z., Ravindran, P.T., Lim, W.A. \& Toettcher, J.E. Mol. Cell 67, 757-769 (2017)
This article was submitted to Nature Methods by a commercial organization and has not been peer reviewed. Nature Methods takes no responsibility for the accuracy or otherwise of the information provided. 